\title{
Wideband and Reconfigurable Vector Antenna using Radiation Pattern Diversity for 3-D Direction-of-Arrival Estimation
}

\author{
Johan Duplouy* ${ }^{* \ddagger}$, Christophe Morlaas* ${ }^{* \ddagger}$, Hervé Aubert ${ }^{\dagger \ddagger}$, Senior Member, IEEE, Patrick Potier ${ }^{\S}$, \\ and Philippe Pouliguen ${ }^{\S}$,
}

\begin{abstract}
The direction finding performances of a novel passive, wideband and radiation pattern reconfigurable vector antenna are reported. Accurate estimation of the direction of arrival of incoming electromagnetic fields across the 3-D half-space is obtained over a 1.69:1 impedance bandwidth from the radiation pattern reconfigurability of a 4-port vector antenna. This antenna consists of only two orthogonal and co-located semi-circular arrays of Vivaldi antennas. Due to its reconfigurability, more radiation patterns can be used in addition to those commonly employed in standard vector antennas to estimate the direction of arrival of incoming electromagnetic fields. A new method based on the Cramer-Rao lower bound is proposed in order to select the radiation pattern diversity that improves the estimation accuracy across the overall bandwidth. Measured and simulated results are found in good agreement. The present study brings a further step towards developing new concepts of reconfigurable vector antennas.
\end{abstract}

Index Terms - wideband magnetic and electric dipoles, Vivaldi antennas, vector sensor, direction-of-arrival antenna, reconfigurable antenna, radiation pattern diversity, direction-of-arrival estimation.

\section{INTRODUCTION}

$\mathbf{E}$ STIMATION of the Direction-of-Arrival (DoA) of incoming ElectroMagnetic (EM) fields plays a crucial role in various civilian and military applications for radionavigation or radio-localization purposes [1]. The key component of the Direction Finding (DF) system is the antenna as its electrical characteristics define the estimation accuracy along with the spatial and frequency coverage. Most of commercially available or published DF antennas operate in the VHF or UHF band and offer a 2-D coverage [2]-[6] (i.e., only the azimuth angle of the DoA can be estimated for a limited range of elevation angles).

Many methods can be applied to estimate the DoA of incoming EM fields. The standard technique is based on the spatial distribution of an antenna array [7]. However, the extension of this technique for wideband DF system targeting 3-D coverage (i.e., estimation of both azimuth and elevation angles of incoming EM fields) is not straightforward, as the spatial and frequency coverage is limited by the spacing between the antennas constituting the array. The use of a compact,

* Ecole Nationale de l'Aviation Civile, TELECOM-EMA, F-31055 Toulouse, France (e-mail: johan.duplouy@enac.fr).

$\uparrow$ LAAS-CNRS, Micro and Nanosystems for Wireless Communications Research Group, F-31055 Toulouse, France.

$\ddagger$ Toulouse University, F-31400 Toulouse, France.

$\S$ Direction Général de l'Armement (DGA), F-75509 Paris, France. low profile and wideband DF antenna with a 3-D field of view is actually very challenging. An innovative technique consists of deriving the DoA from the measurement of the six components of the EM field through a so-called Vector Antenna (VA). Ferrara and Parks introduced the concept of diversely polarized DF antennas in 1983 by using only electric dipoles with orthogonal orientation [8]. Hatke developed in 1992 the SuperCART antenna, consisting of three electric dipoles and three magnetic dipoles, all spatially co-located with orthogonal orientations, which is the cornerstone of the VA design [9]. Then, Nehorai and Paldi formally extended this concept in 1994 by measuring both electric and magnetic information to estimate the DoA of an incident EM field [10]. VAs are gaining prominence since their introduction due to the three following reasons: $(i)$ a single VA is sufficient to estimate the DoA with 3-D coverage; (ii) as they may replace large antenna arrays, VAs enable high mobility thanks to their compactness; (iii) the discrimination of two close directions of incoming EM fields can be performed from polarization difference [11].

Many VA designs have been reported for estimating the DoA of incoming EM fields [12]-[17]. However, only few designs demonstrated the capability of covering the full 3-D space [15]-[17]. Moreover, the attention was mainly focused on the implementation of active VA structures. An active solution aimed at covering frequencies below $30 \mathrm{MHz}$ was patented in 2013 [15]. Another interesting active solution combining three small loops and operating either as an electric dipole or as a magnetic dipole from excitation control has been reported in [16]. However, active antennas exhibit strong limitations, such as: (i) a power supply device is required to feed the active elements; (ii) the use of active devices may lead to harmonics generation, signal distortion, etc. Fully passive VAs do not suffer from these limitations, but their wideband coverage is very challenging to achieve. A passive VA operating in two separate narrow bandwidths was proposed in [17] and very recently, we reported in [18] the first passive, wideband and Radiation Pattern (RP) reconfigurable VA. However, the DF performances of this antenna were not reported. The antenna exhibits a 1.69:1 bandwidth from $2.1 \mathrm{GHz}$ to 3.55 $\mathrm{GHz}$, and it can be used for aeronautical navigation as well as for mobile or WLAN location applications. This VA consists of two spatially co-located and orthogonally oriented dual-port semi-circular arrays of Vivaldi antennas, and is referred to as the Two Season VA in the remainder of this paper. Besides, 
the antenna is included in a half-sphere within a $0.52 \lambda_{0}$ radius and mounted on a finite metallic and octagonal support with a radius of $1.14 \lambda_{0}$, where $\lambda_{0}$ is the free space wavelength at the lowest operating frequency. Up to now, and to the best authors' knowledge, no wideband and passive VA has proven its capability of covering the 3-D space, since previous works on wideband VAs were focused on active structures.

In this paper, the DF performances of the first passive and wideband VA designed to cover the 3-D upper halfspace are assessed in detail, including the evaluation of the prototype sensitivity for DoA estimation errors below $5^{\circ}$. For the first time, the DF performances of VAs are enhanced across the overall bandwidth thanks to a new DF technique consisting of adding more RPs to those commonly used for the DoA estimation. The appropriate additional RPs are selected by an original method based on the Cramer-Rao lower Bound (CRB). Although its benefits are demonstrated here by evaluating the simulated and measured DF performances of the Two Season VA, the RP diversity technique could be advantageously applied to any VAs with reconfigurable RP.

This paper is organized as follows. Section II reports the DF principle using RP diversity for RP reconfigurable VAs. The observation model used in the DoA estimation process is also described. Section III reports the simulated and measured DF performances of the reconfigurable Two Season VA using only the measurement of three components of the incoming EM field. Section IV presents an efficient method based on the CRB for selecting the RPs that improve the DoA estimation accuracy. Section V analyzes the simulated and measured DF performances when the optimal additional RPs are used for the DoA estimation. Conclusion and future directions are drawn in Section VI. The definition of descriptors used here to assess the DF performances is given in Appendix A.

\section{Direction Finding TeChnique Based on RADIATION PATTERN DIVERSITY OF VECTOR ANTENNAS}

This section describes the DF principle based on RP diversity for RP reconfigurable VAs along with the observation model used in the DoA estimation process.

\section{A. DF principle}

In principle, the angles of arrival $\theta$ (elevation angle) and $\phi$ (azimuth angle) of an incoming EM field can be derived from the measurement at the VA location of the six components of this field, said $E_{x}, E_{y}, E_{z}, H_{x}, H_{y}$ and $H_{z}$ in the Cartesian coordinate system (refer to Fig. 1 for its definition). Two main approaches allow the measurement of these field components:

- The first one consists of using six orthogonally oriented electric and magnetic dipoles, which are all spatially located in a point-like geometry [19]. In this well-known approach, every EM field component is derived from the signal received by a specific constitutive dipole. Indeed, the measurement of a given electric or magnetic field component is associated with the RP of an electric or magnetic dipole. For instance, the component $E_{x}$ is derived from the signal received by an electric dipole oriented along $\hat{x}$. This signal is obtained thanks to the RP of this specific dipole.

- The second approach consists of using radiating elements other than electric or magnetic dipole [16]. Every EM field component is then derived from the appropriate combination or weighted summation of signals simultaneously received by the relevant constitutive radiating elements. This approach is valid as long as the weighting coefficients assigned to the received signals enable the synthesis of the RPs of three electric dipoles and three magnetic dipoles. In this approach, the VA can be viewed as a RP reconfigurable VA. In practice, the set of weighting coefficients assigned to the received signals for measuring every EM field component can be obtained from either passive circuits or signal post-processing.

Once all the EM field components are obtained from applying one of these two main approaches, high-resolution algorithms, such as MUSIC (MUltiple SIgnal Classification) [20], can then be applied to derive an estimation of the DoA.

In this paper, it will be shown that using more RPs (or accordingly, more sets of weighting coefficients assigned to each received signal) in addition to those commonly used in standard VAs may improve the DoA estimation accuracy across the overall bandwidth. A method based on the CRB will be proposed in Section IV for selecting these additional RPs.

\section{B. Observation model}

1) General case: Let $F$ be the number of narrow-band and EM plane waves traveling in an isotropic, homogeneous and lossless medium and incident upon a $P$-port VA placed at the origin of the Cartesian coordinate system. As depicted in Fig. 1, the VA comprises three orthogonal electic dipoles and three orthogonal electric loops in order to measure the three components of the electric field and the three components of the magnetic field, respectively. The DoA of the $f^{\text {th }}$ incoming EM field is described in the 3-D space by the direction $\left(\phi_{f}, \theta_{f}\right)$ of the wave vector $\boldsymbol{k}$, where $\phi_{f} \in\left[0^{\circ} ; 360^{\circ}\right]$ and $\theta_{f} \in\left[0^{\circ} ; 180^{\circ}\right]$ denote the azimuth and elevation angles, respectively. Furthermore, the polarization of the $f^{\text {th }}$ incoming EM field is here described by the polarization vector $\boldsymbol{p}_{f}$ (referred sometimes as the Jones vector) and is defined as the combination of vertical $\left(\boldsymbol{p}_{\boldsymbol{V}}\right)$ and horizontal $\left(\boldsymbol{p}_{\boldsymbol{H}}\right)$ polarizations as follows

$$
\boldsymbol{p}_{f}=\sin \left(\gamma_{f}\right) e^{j \eta_{f}} \boldsymbol{p}_{\boldsymbol{V}}+\cos \left(\gamma_{f}\right) \boldsymbol{p}_{\boldsymbol{H}},
$$

where $\gamma_{f} \in\left[0^{\circ} ; 90^{\circ}\right]$ and $\eta_{f} \in\left[-90^{\circ} ; 90^{\circ}\right]$ refer to the auxiliary polarization angle and the polarization phase difference, respectively. Therefore, the parameters of the $f^{\text {th }}$ incoming EM field can be summarized by the vector parameter $\boldsymbol{\Omega}_{f}=\left[\begin{array}{llll}\theta_{f} & \phi_{f} & \eta_{f} & \gamma_{f}\end{array}\right]^{\mathrm{T}}$.

It is assumed here that $M$ different sets of weighting coefficients can be assigned to the signals received at the $P$ ports of the VA with the help of a passive circuit or signal post-processing. The $M$ available RPs include those allowing the measurement of the components of incoming EM fields. For $F$ EM fields incident upon the VA, the voltage $\boldsymbol{x} \in \mathbb{C}^{P, 1}$ 


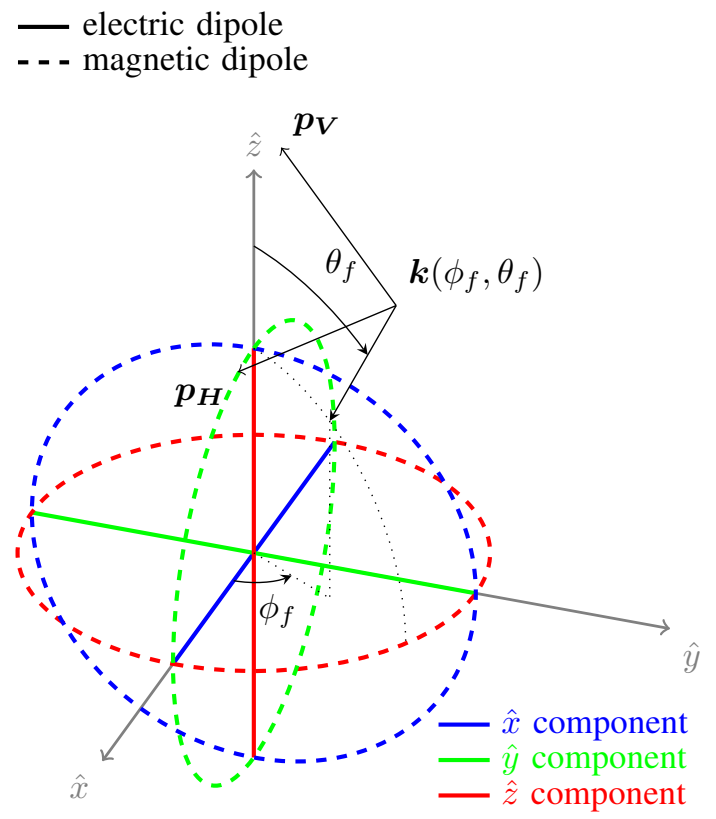

Fig. 1: Topology of a typical VA that enables the measurement of the six components of the $f^{\text {th }}$ incident EM field in the Cartesian coordinate system. Its DoA is defined by the azimuth $\phi_{f}$ and elevation $\theta_{f}$ angles.

received at time $t$ and at the $P$ ports of the VA can be written as follows

$$
\boldsymbol{x}(t)=\boldsymbol{d}(\phi, \theta, \gamma, \eta) \cdot \boldsymbol{s}(t)+\boldsymbol{n}(t)
$$

where $\boldsymbol{d} \in \mathbb{C}^{P, F}$ is the VA response which includes the polarization vector $\boldsymbol{p}$. Moreover, $s \in \mathbb{C}^{F, 1}$ designates the signal vector associated with the $F$ incoming EM fields at time $t$ and characterized by $\boldsymbol{\Omega}_{f}$. In Eq. (2), $\boldsymbol{n} \in \mathbb{C}^{P, 1}$ denotes the additive white Gaussian noise. It is assumed here that this noise is spatially invariant with zero-mean and covariance matrix $\boldsymbol{R}_{\boldsymbol{n}}=\sigma_{n}^{2} \boldsymbol{I} \in \mathbb{C}^{P, P}$. The combined data model $\boldsymbol{x}_{\boldsymbol{c}} \in \mathbb{C}^{M, 1}$ at time $t$ is then derived from the summation of received signals after assigning to every signal an appropriate weighting coefficient. The $m^{\text {th }}$ component of $\boldsymbol{x}_{\boldsymbol{c}}$ associated with the $m^{\text {th }}$ set can be written as follows

$$
x_{c}^{m}(t)=\boldsymbol{\beta}^{m} \cdot \boldsymbol{x}(t),
$$

where $\boldsymbol{\beta}^{m}=\left[\beta_{1}^{m} \ldots \beta_{p}^{m} \ldots \beta_{P}^{m}\right] \in \mathbb{C}^{1, P}$ denotes the complex weighting coefficients applied at the $P$ ports of the VA (and corresponds to the $m^{\text {th }} \mathrm{RP}$ ). Usually, a set of $N$ snapshots is used for the DoA estimation and thereby, the observation model can be written as $\boldsymbol{X}_{\boldsymbol{c}}=\left[x_{c}(1), x_{c}(2), \ldots, x_{c}(N)\right] \in$ $\mathbb{C}^{M, N}$

2) Application to the DoA estimation of vertically polarized EM fields in the 3-D upper half-space: The addition of new RPs can be applied for the DoA estimation of incoming EM fields, even if the VA enables the measurement of less than six EM field components. As shown in [17], the measurement of only $E_{z}, H_{x}$ and $H_{y}$ is sufficient for estimating the DoA of incoming vertically polarized EM fields $(\gamma=\pi / 2$ and $\eta=0$ ). This is also the case for the Two Season VA depicted in Fig. 2. This antenna enables the measurement of these three components in the 3-D upper half-space (i.e., $\phi_{f} \in\left[0^{\circ} ; 360^{\circ}\right]$ and $\left.\theta_{f} \in\left[0^{\circ} ; 90^{\circ}\right]\right)$. The amplitude and phase of the received signal are sequentially measured at each port, while the other ports are impedance matched. Next, each EM field component is derived from the summation of signals that are received at the four ports of the antenna $(P=4)$, after assigning to every signal an appropriate weighting $\boldsymbol{\beta}^{m}$ (one weight per port). Hence, a set of four weights is assigned to the four ports of the VA to measure one EM component. This set constitute a specific RP. Several RPs are grouped to define a RPC (Radiation Patterns Combination). Table I reports the three sets of weighting coefficients assigned to the received signals at the antenna ports for deriving the three EM field components (denoted by RPC 0 in the remainder of this paper).
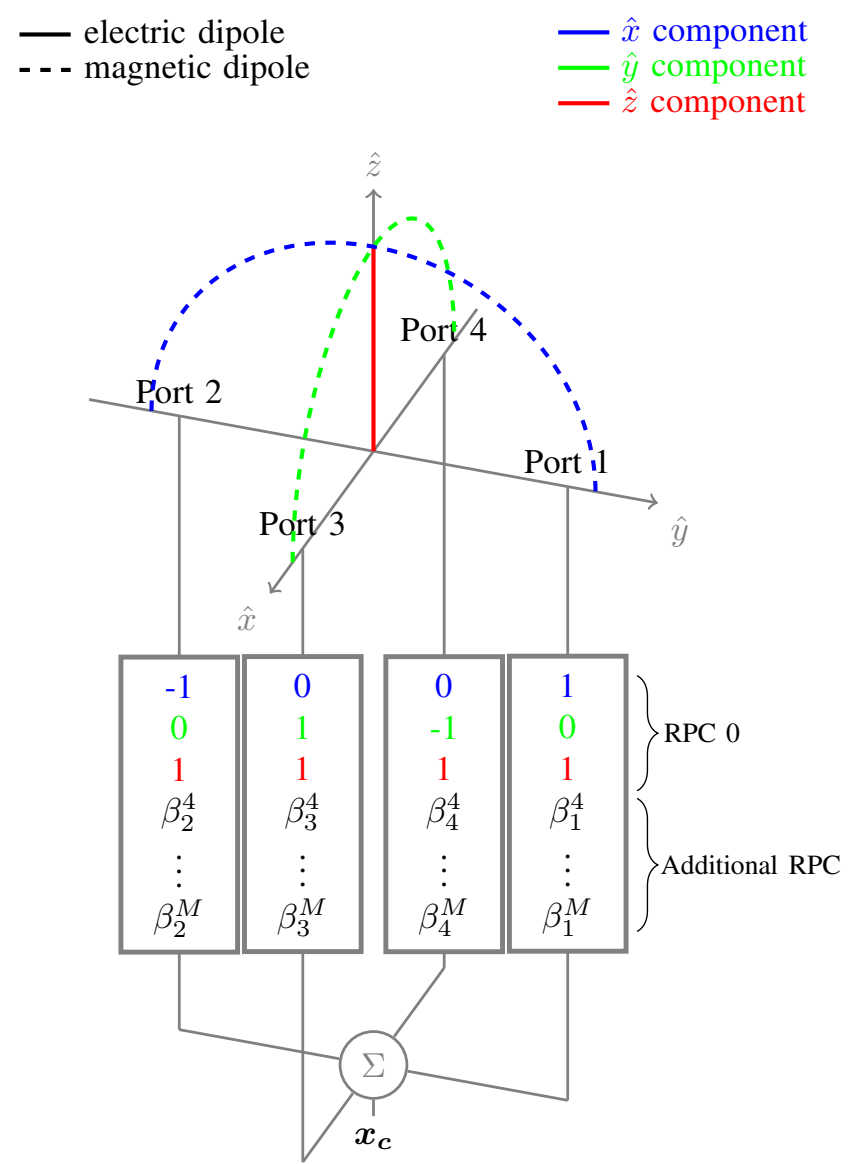

Fig. 2: Sets of weighting coefficients applied to the four ports of the Two Season VA for measuring the EM field components $E_{z}, H_{x}$ and $H_{y}$ (RPC 0) and for the additional RP diversity (see Section IV). 
TABLE I: Three sets of weighting coefficients $\boldsymbol{\beta}=$ $\left[\begin{array}{llll}\beta_{1} & \beta_{2} & \beta_{3} & \beta_{4}\end{array}\right]$ assigned to the signals received at the four ports of the Two Season VA for measuring the three EM field components $H_{x}, H_{y}$ and $E_{z}$ (corresponding to RPC 0)

\begin{tabular}{cccccc} 
RPC & $\begin{array}{c}\text { Measured } \\
\text { Component }\end{array}$ & $\begin{array}{c}\text { Port } 1 \\
\beta_{1}\end{array}$ & $\begin{array}{c}\text { Port 2 } \\
\beta_{2}\end{array}$ & $\begin{array}{c}\text { Port 3 } \\
\beta_{3}\end{array}$ & $\begin{array}{c}\text { Port } 4 \\
\beta_{4}\end{array}$ \\
\hline \multirow{3}{*}{0} & $H_{x}$ & 1 & -1 & 0 & 0 \\
& $H_{y}$ & 0 & 0 & 1 & -1 \\
& $E_{z}$ & 1 & 1 & 1 & 1 \\
\hline
\end{tabular}

For the sake of simplicity, we assume in this paper that only one vertically polarized EM field is incident upon the VA $(F=1)$. The DF performances evaluation is based on the standard MUSIC algorithm (MUltiple SIgnal Classification) [20] applied to our observation model. Last but not least, as some amplitude and phase distortions may eventually occur at the VA outputs, a calibration process (see, e.g., [1], [21]) is generally applied in practice. The calibration technique applied here is based on the computation of the combined steering vector $\boldsymbol{d}$ from the simulated or measured RPs of the antenna VA radiating elements as described in [17]. Although we use the aforementioned VA for demonstrating the proofof-concept, the proposed method can be applied to any VAs.

\section{Direction Finding PERformances of THE RECONFIGURABLE VECTOR ANTENNA WITHOUT APPLYING THE PROPOSED RADIATION PATTERN DIVERSITY TECHNIQUE}

In this section, the simulated and measured DF performances of the reconfigurable wideband Two Season VA (depicted in Fig. 3) are compared and discussed. Only the three sets, reported in Table I, of weighting coefficients assigned to signals received at the four antenna ports are used here for measuring the EM field component $H_{x}, H_{y}$ and $E_{z}$ (RPC 0).

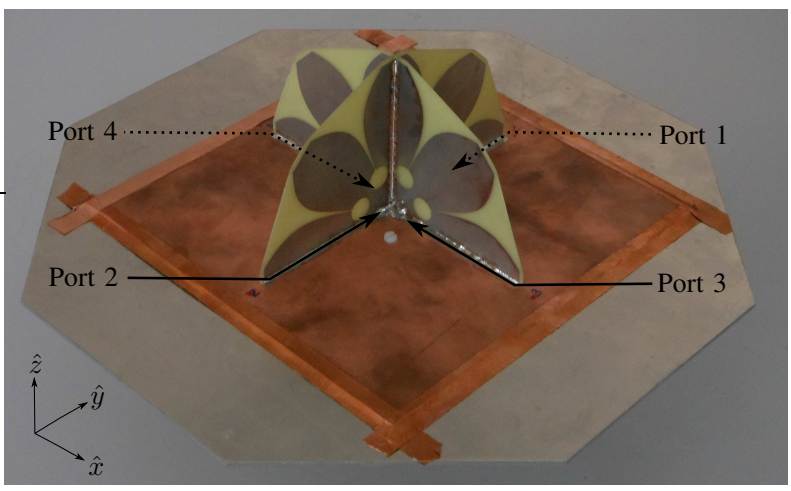

Fig. 3: Photograph of the 4-port Two Season VA [18]

The DF performances are given at three frequencies 2.2 $\mathrm{GHz}, 2.8 \mathrm{GHz}$, and $3.4 \mathrm{GHz}$, which are respectively, the lower, center and upper frequencies of the Two Season VA operating bandwidth. The DoAs are computed from the MUSIC algorithm and full-wave EM simulation (HFSS software) or measurement data. All estimation parameters are reported in Table II. At the VA location, the power density of the incident field is of $-105 \mathrm{dBW} \cdot \mathrm{m}^{-2}$. Moreover, the noise floor power level at the receiver output is of $-111 \mathrm{dBm}$. Consequently, the DF performances evaluation is carried out for a power density-tonoise ratio (PDNR) of $36 \mathrm{~dB} \cdot \mathrm{m}^{-2}$, defined more precisely in Appendix A-A. Furthermore, following [17], the analysis is performed for $N=100$ snapshots per DoA estimation, and the number $L$ of Monte Carlo trials is set to 20 .

TABLE II: Parameters used for evaluating the DF performances

\begin{tabular}{lc}
\hline DoA Algorithm & MUSIC \\
\hline Number of incoming EM fields & 1 \\
\hline Polarization of the incoming EM field & Vertical \\
\hline Angular coverage & $\begin{array}{c}\phi \in\left[0^{\circ} ; 90^{\circ}\right] \\
\theta \in\left[0^{\circ} ; 90^{\circ}\right]\end{array}$ \\
\hline Angular resolution & $\Delta \phi=5^{\circ}$ \\
& $\Delta \theta=2^{\circ}$ \\
\hline Incoming EM field power density & $-105 \mathrm{dBW} \cdot \mathrm{m}^{-2}$ \\
\hline Noise power level & $-111 \mathrm{dBm}$ \\
\hline PDNR & $36 \mathrm{~dB} \cdot \mathrm{m}^{-2}$ \\
\hline Snapshots per DoA estimation & 100 \\
\hline Number of estimations per DoA & 20 \\
\hline & $2.2 \mathrm{GHz}$ \\
Frequencies of interest & $2.8 \mathrm{GHz}$ \\
& $3.4 \mathrm{GHz}$ \\
\hline
\end{tabular}

Fig. 7 (columns 1 and 2) displays the simulated and measured DoA estimation accuracy specified by the angular distance $\Delta a_{\mathrm{RMS}}$ (defined in Appendix A-D). Globally, measurement and simulation results are in good agreement. It can be observed that $\Delta a_{\mathrm{RMS}}$ does not exceed $5^{\circ}$ for almost every direction of the incoming EM field within the antenna bandwidth. Aside from some particular directions, the larger values of simulated and measured $\Delta a_{\mathrm{RMS}}$ are obtained in same angular areas which depend on the operating frequency. These results can be justified by analyzing on the one hand, the CRB (in $\theta$ and in $\phi$ ) which specifies the smallest achievable estimation errors (see Appendix A-B) and, on the other hand, the 3D ambiguity spectrum which indicates the directions in which the risk of angular ambiguity is high (see Appendix A-C). The analysis of these results is conducted in Section V-B. However, it is very challenging to compare the accuracy of the DoA estimate from one direction to another because: (i) undesirable RP distortion may occur due to inevitable technological imprecision in the VA manufacturing; (ii) very isolated errors can be missed due to a slight angular offset (the chosen azimuthal angular step is of $5^{\circ}$ ); (iii) gears in the positioning system may be inaccurate. Indeed, the measured estimation accuracy is slightly degraded at all frequencies since errors appear only for elevation angles close to $0^{\circ}$ and are not present in the simulation results. The results of the DoA estimation for simulation and measurement are summarized in Table VI, where the maximum as well as the $95^{\text {th }}$ percentile 
of $\Delta a_{\mathrm{RMS}}$ are reported. As above-discussed, a discrepancy between the simulated and measured maximum values of $\Delta a_{\mathrm{RMS}}$ is observable. However, at the three frequencies of interest, the simulated and measured $95^{\text {th }}$ percentile of the $\Delta a_{\mathrm{RMS}}$ is lower than $2.0^{\circ}$ and $3.1^{\circ}$, respectively. Finally, these estimations of the DoA obtained from the first passive and wideband VA are very encouraging, and they allow the experimental validation of the simulated DF performances.

\section{Methodology for Selecting the Radiation PATTERN DIVERSITY AND SIMULATED DiRECTION FINDING PERFORMANCES}

As indicated in Table I, the proposed reconfigurable VA enables the measurement of the three components $E_{z}, H_{x}$ and $H_{y}$ of the incoming vertically polarized EM field through three sets ${ }^{1}$ (denoted by RPC 0) of weighting coefficients assigned to the signals received at the four antenna ports. Now, we propose to add more sets of weighting coefficients (or accordingly more RPs) to eventually improve the DoA estimation. This section presents the methodology used for selecting the additional RP diversity and analyzes the accuracy of DoA estimation acccording to the choice of the RPs.

\section{A. Criterion for selecting the additional RPs based on the $C R B$}

1) Methodology: By assigning random weighting coefficients to the signals received at the 4-port VA, a large number of sets may be added to the three initial ones used in the previous DoA estimation process (see RPC 0 in Section III). Let 1, 0 and -1 be the possible values for the weighting coefficients. As a result, 81 sets of weighting coefficients could a priori be assigned to the signals received at the four ports of the Two Season VA. Some of these sets of weighting coefficients (such as, e.g., the set $[1,1,-1,1]$ and the set $[-1,-1,1,-1])$ provide identical RPs when used as excitation laws for feeding the four ports of the Two Season VA. In these cases, only one set is then kept for the DoA estimation, while the others are discarded. Therefore, it remains 37 different RPs and consequently, there $\operatorname{are}^{2} \sum_{k=1}^{37}\left(\begin{array}{c}37 \\ k\end{array}\right)=2^{37}-1$ available RPCs that can be added to RPC 0 to improve the DF performances. From there, the challenge is to select the RPCs that maximize the DoA estimation accuracy. In order to avoid a long processing time for detecting the RPCs that improve the DF performances, we proceed to a pre-selection of 11 RPCs. Each of these RPCs consists of several sets of weighting coefficients that provide the same RP with a rotation of $90^{\circ}$ in the azimuth plane in order to ensure the same DoA estimation performances across the 3-D half-space. These additional RPCs are given in Table III and include 37 different RPs. To select among them the appropriate RPC which achieves the highest DoA

\footnotetext{
${ }^{1} \mathrm{~A}$ set is defined as $\left[\begin{array}{llll}\beta_{1} & \beta_{2} & \beta_{3} & \beta_{4}\end{array}\right]$, where $\beta_{i} \in \llbracket 1 ; 4 \rrbracket$ designates the weighting coefficient at port $i$.

${ }^{2}$ The acronym RPC means Radiation Patterns Combination and represents a group of RPs. The left side sums the number of RPCs of sizes $k=\{1,2, \ldots, 37\}$ (or equivalently, the left side sums number of RPCs comprising $k$ different RPs). The order of selection of the RPs constituting the RPC does not matter.
}

estimation accuracy in the 3-D half-space, we propose to compute the CRB.

TABLE III: RPC and corresponding weighting coefficients assigned to the signals received by the 4-port Two Season VA

\begin{tabular}{|c|c|c|c|c|c|c|c|c|c|c|c|c|}
\hline \multirow{6}{*}{$\begin{array}{l}\text { Port } 1 \\
\text { Port } 2 \\
\text { Port } 3 \\
\text { Port } 4\end{array}$} & \multicolumn{4}{|c|}{$\mathrm{RPC} 1^{\mathrm{a}}$} & \multicolumn{4}{|c|}{ RPC 2} & \multicolumn{4}{|c|}{ RPC $3^{b}$} \\
\hline & $\mathrm{RP}$ & \multirow{2}{*}{$\begin{array}{l}\mathrm{RP} \\
0\end{array}$} & \multirow{2}{*}{$\begin{array}{l}\mathrm{RP} \\
0\end{array}$} & $\mathrm{RP}$ & \multirow{2}{*}{$\mathrm{RP}$} & \multirow{2}{*}{$\mathrm{RP}$} & \multirow{2}{*}{$\frac{\mathrm{RP}}{1}$} & $\mathrm{RP}$ & \multirow{2}{*}{$\mathrm{RP}$} & RP & & \\
\hline & 1 & & & 0 & & & & 0 & & 0 & & \\
\hline & 0 & 0 & 1 & 0 & 1 & 0 & 1 & 1 & 1 & 0 & & \\
\hline & 0 & 0 & 0 & 1 & 1 & 1 & 0 & 1 & 0 & 1 & & \\
\hline & 0 & 1 & 0 & 0 & 0 & 1 & 1 & 1 & 0 & 1 & & \\
\hline & \multicolumn{4}{|c|}{ RPC 4} & \multicolumn{4}{|c|}{ RPC 5} & \multicolumn{4}{|c|}{ RPC 6} \\
\hline & $\mathrm{RP}$ & $\mathrm{RP}$ & $\mathrm{RP}$ & $\mathrm{RP}$ & $\mathrm{RP}$ & $\mathrm{RP}$ & $\mathrm{RP}$ & $\mathrm{RP}$ & $\mathrm{RP}$ & $\mathrm{RP}$ & $\mathrm{RP}$ & $\mathrm{RP}$ \\
\hline Port 1 & 1 & 1 & 1 & -1 & 1 & 0 & 1 & -1 & 1 & 1 & 0 & 0 \\
\hline Port 2 & 1 & -1 & 1 & 1 & 1 & -1 & 1 & 0 & 0 & 0 & 1 & 1 \\
\hline Port 3 & 1 & 1 & -1 & 1 & 0 & 1 & -1 & 1 & 1 & 0 & 0 & 1 \\
\hline \multirow[t]{3}{*}{ Port 4} & -1 & 1 & 1 & 1 & -1 & 1 & 0 & 1 & 0 & 1 & 1 & 0 \\
\hline & \multicolumn{4}{|c|}{$\operatorname{RPC} 7^{b}$} & \multicolumn{4}{|c|}{ RPC 8} & \multicolumn{4}{|c|}{ RPC $9^{b}$} \\
\hline & $\mathrm{RP}$ & & & & $\mathrm{RP}$ & $\mathrm{RP}$ & $\mathrm{RP}$ & $\mathrm{RP}$ & $\mathrm{RP}$ & $\mathrm{RP}$ & & \\
\hline Port 1 & 1 & & & & 1 & 0 & 0 & -1 & 1 & 1 & & \\
\hline Port 2 & 1 & & & & 0 & -1 & 1 & 0 & -1 & -1 & & \\
\hline Port 3 & -1 & & & & 0 & 0 & -1 & 1 & 1 & -1 & & \\
\hline \multirow[t]{3}{*}{ Port 4} & -1 & & & & -1 & 1 & 0 & 0 & -1 & 1 & & \\
\hline & \multicolumn{4}{|c|}{ RPC 10} & \multicolumn{4}{|c|}{ RPC 11} & & & & \\
\hline & $\mathrm{RP}$ & $\mathrm{RP}$ & $\mathrm{RP}$ & $\mathrm{RP}$ & $\mathrm{RP}$ & $\mathrm{RP}$ & $\mathrm{RP}$ & $\mathrm{RP}$ & & & & \\
\hline Port 1 & 1 & 1 & 0 & $\overline{-1}$ & 1 & 1 & 0 & $\overline{-1}$ & & & & \\
\hline Port 2 & 0 & -1 & 1 & 1 & 0 & -1 & 1 & 1 & & & & \\
\hline Port 3 & 1 & 0 & -1 & 1 & -1 & 1 & 1 & 0 & & & & \\
\hline Port 4 & -1 & 1 & 1 & 0 & 1 & 0 & -1 & 1 & & & & \\
\hline
\end{tabular}

2) Selection of the additional RPC: For each RPC, the $\mathrm{CRB}_{\theta}$ and $\mathrm{CRB}_{\phi}$ are computed from the parameters displayed in Table IV at the three frequencies and using the simulated RPs of the Two Season VA.

TABLE IV: Parameters used for the CRB analysis

\begin{tabular}{lc}
\hline Number of incoming EM fields & 1 \\
\hline Polarization of the incoming EM field & Vertical \\
\hline Angular coverage & $\phi \in\left[0^{\circ} ; 360^{\circ}\right]$ \\
$\theta \in\left[0^{\circ} ; 90^{\circ}\right]$ \\
\hline Angular resolution & $\Delta \phi=1^{\circ}$ \\
& $\Delta \theta=1^{\circ}$ \\
\hline Incoming EM field power density & $-117 \mathrm{dBW} \cdot \mathrm{m}^{-2}$ \\
\hline Noise power level & $-111 \mathrm{dBm}$ \\
\hline PDNR & $24 \mathrm{~dB} \cdot \mathrm{m}^{-2}$ \\
\hline Snapshots per DoA estimation & 100 \\
\hline & $2.2 \mathrm{GHz}$ \\
\hline
\end{tabular}

The CRB corresponds to the smaller estimation error achievable by the antenna regardless of the applied DF technique. Therefore, selecting the additional RPC with respect to the smallest value of CRB ensures the best DoA estimation performances. In this paper, the optimal RPC is defined as 
the RPC $i(i \in \llbracket 1 ; 11 \rrbracket)$ to be added to RPC 0 for minimizing the average of the maximum $\mathrm{CRB}_{\theta}$ and $\mathrm{CRB}_{\phi}$ computed for the frequencies of interest in the VA bandwidth. The analysis of $\mathrm{CRB}_{\theta}$ and $\mathrm{CRB}_{\phi}$ is illustrated in Figs. 4 and 5, respectively. It can be observed that when only RPC 0 is used, the estimation errors are mainly found on $\phi$ rather than $\theta$, as the average $\mathrm{CRB}_{\phi}\left(0.4^{\circ}\right)$ is significantly higher than $\mathrm{CRB}_{\theta}\left(5.9^{\circ} \times 10^{-3}\right)$. Moreover, it can be observed that the different RPCs are more or less effective in improving the DoA estimation accuracy in azimuth or elevation. The average CRB (diamond marks) indicates that RPC 4 is the optimal combination. Futhermore, RPC 4 achieves practically the highest accuracy for estimating the DoA (in azimuth and elevation) at the three frequencies of interest. Finally, the best second candidate for all frequencies and for $\phi$ and $\theta$ is RPC 10 and corresponds to the average of the maximum $\mathrm{CRB}_{\phi}$ and $\mathrm{CRB}_{\theta}$ of $6.8^{\circ} \times 10^{-3}$ and $2.3^{\circ} \times 10^{-3}$ (instead of $5.5^{\circ} \times 10^{-3}$ and $1.5^{\circ} \times 10^{-3}$ with RPC 4).

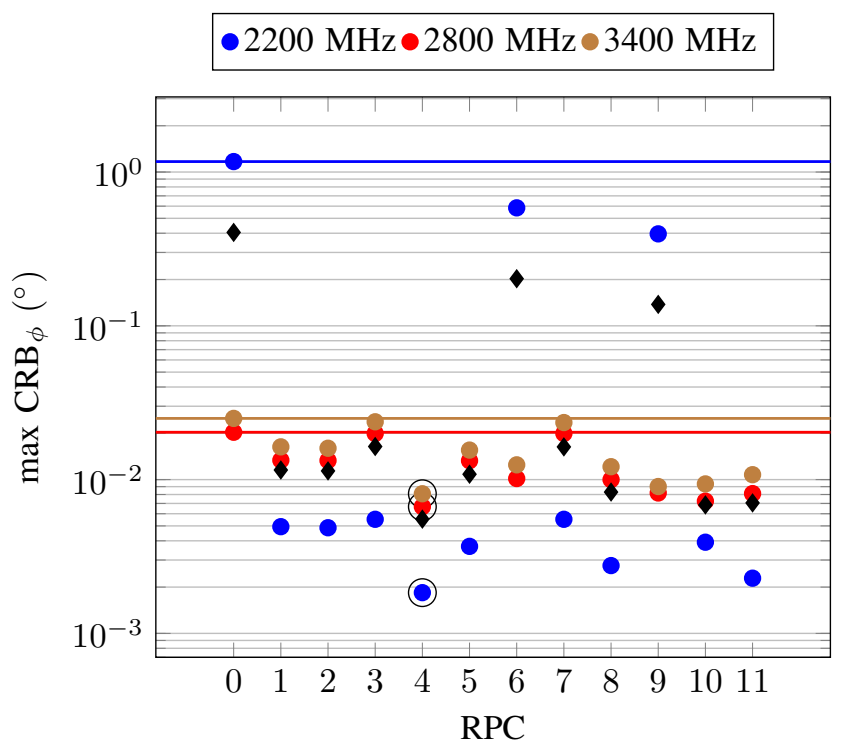

Fig. 4: Highest simulated $\mathrm{CRB}_{\phi}$ using different RPCs [1 to 11] added to the RPC 0 , and under the scenario conditions defined in Table IV at $2.2 \mathrm{GHz}, 2.8 \mathrm{GHz}$ and $3.4 \mathrm{GHz}$. The horizontal lines and black circles correspond to the reference maximum CRB (obtained using RPC 0 only) and the lowest maximum $\mathrm{CRB}$ value, respectively. The black diamonds denote the average maximum $\mathrm{CRB}$ for all three frequencies.

\section{B. DoA estimation results from MUSIC analysis}

In order to validate the selection of the RPC based on the CRB, DoA estimation performances of the reconfigurable Two Season VA are evaluated here through the MUSIC algorithm. Only simulated RPs are used in this section. The parameters used for evaluating the DF performances are similar to those reported in Table II, except that the PDNR is decreased by $12 \mathrm{~dB}$ with respect to the PDNR given in Section III. The incoming power density is set to $-117 \mathrm{dBW} \cdot \mathrm{m}^{-2}(\mathrm{PDNR}=24$ $\mathrm{dB} \cdot \mathrm{m}^{-2}$ ) in order to highlight the benefits of adding RP diversity in the DoA estimation process. For comparison purpose, Table $\mathrm{V}$ reports the maximum as well as the $95^{\text {th }}$ percentile

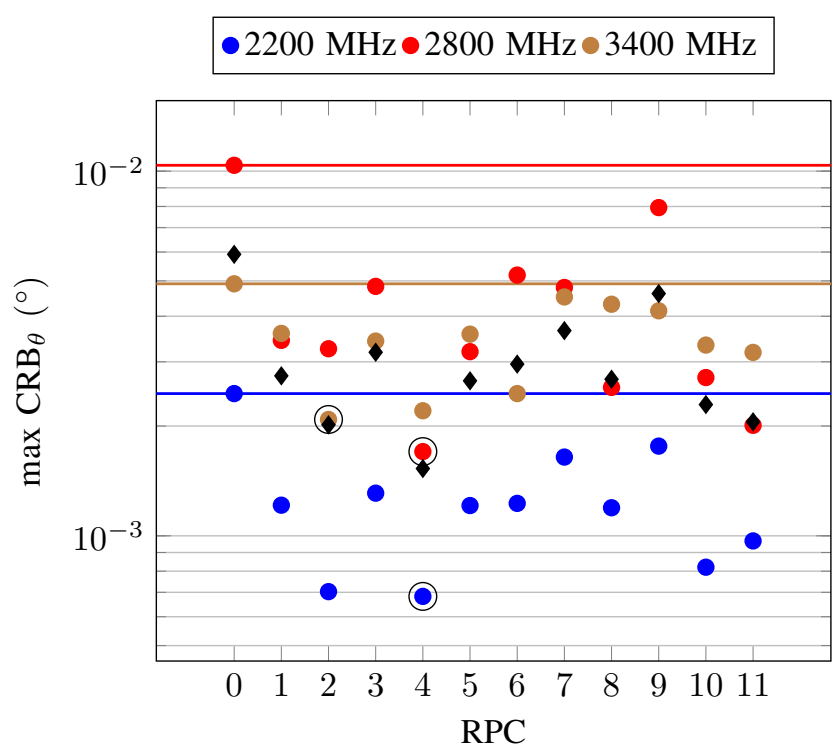

Fig. 5: Highest simulated $\mathrm{CRB}_{\theta}$ using different RPCs [1 to 11] added to the RPC 0 , and under the scenario conditions defined in Table IV at $2.2 \mathrm{GHz}, 2.8 \mathrm{GHz}$ and $3.4 \mathrm{GHz}$. The horizontal lines and black circles correspond to the reference maximum CRB (obtained using RPC 0 only) and the lowest maximum CRB value, respectively. The black diamonds denote the average maximum CRB for all three frequencies.

of $\Delta a_{\mathrm{RMS}}$. As expected, since the PDNR is significantly lower, the simulated DF performances of the Two Season VA achieved using the measurement of only three components of the incoming EM field (RPC 0) is significantly degraded compared with that reported in Table VI.

TABLE V: Simulated DF performances from using RPC 0 only, and under the scenario conditions defined in Table II (except that PDNR=24 dB.m ${ }^{-2}$ )

\begin{tabular}{|c|c|c|c|c|}
\hline \multirow[b]{2}{*}{ Frequency } & \multicolumn{2}{|c|}{ RPC 0} & \multicolumn{2}{|c|}{ RPC 4} \\
\hline & $\begin{array}{c}\max \\
\Delta a_{\mathrm{RMS}}\end{array}$ & $\begin{array}{c}95^{\text {th }} \text { percentile } \\
\text { of } \Delta a_{\mathrm{RMS}}\end{array}$ & $\begin{array}{c}\max \\
\Delta a_{\mathrm{RMS}}\end{array}$ & $\begin{array}{c}95^{\text {th }} \text { percentile } \\
\text { of } \Delta a_{\mathrm{RMS}}\end{array}$ \\
\hline $2.2 \mathrm{GHz}$ & $15.1^{\circ}$ & $8.2^{\circ}$ & $4.8^{\circ}$ & $3.3^{\circ}$ \\
\hline $2.8 \mathrm{GHz}$ & $33.5^{\circ}$ & $15.0^{\circ}$ & $21.6^{\circ}$ & $5.3^{\circ}$ \\
\hline $3.4 \mathrm{GHz}$ & $140.3^{\circ}$ & $74.6^{\circ}$ & $7.8^{\circ}$ & $3.7^{\circ}$ \\
\hline
\end{tabular}

For each RPC, the simulated $95^{\text {th }}$ percentile of $\Delta a_{\mathrm{RMS}}$ is displayed in Fig. 6. As predicted from the CRB analysis (see Section IV-A), RPC 4 achieves the highest level of accuracy over the VA bandwidth. Using this RPC in addition to RPC 0 enables the reduction of the $95^{\text {th }}$ percentile of $\Delta a_{\mathrm{RMS}}$ by the factors 2.5 at $2.2 \mathrm{GHz}, 2.8$ at $2.8 \mathrm{GHz}$ and 20 at $3.4 \mathrm{GHz}$. It is only reduced by the factors $1.9,2.3$ and 16.6 at these frequencies if RPC 10 is used. Overall, these results confirm the interest of using the CRB for rapidly selecting the RPC to be added to RPC 0 . 


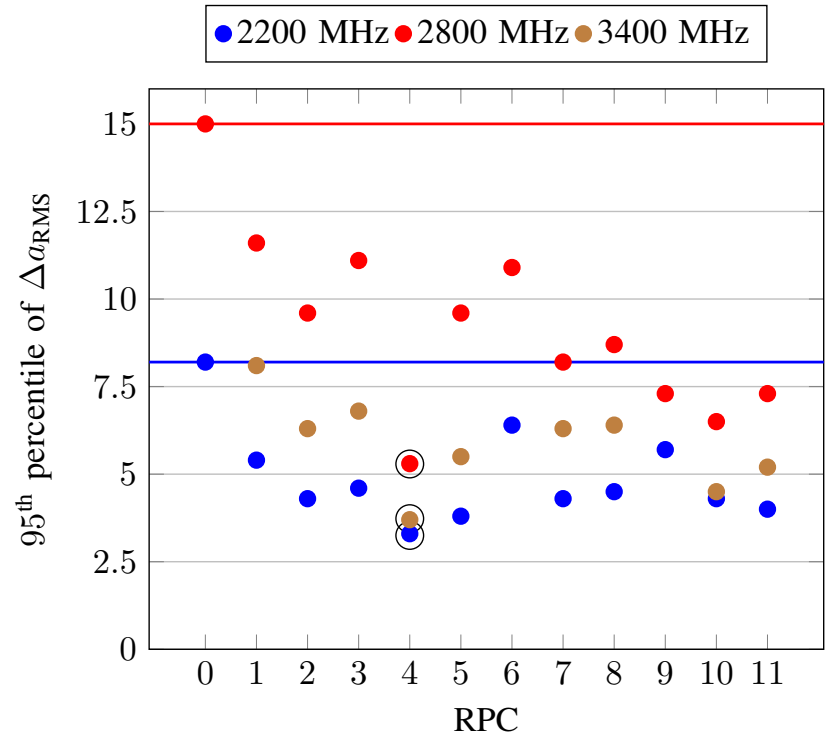

Fig. 6: Simulated $95^{\text {th }}$ percentile of $\Delta a_{\text {RMS }}$ obtained from the reconfigurable VA adding different RPCs [1 to 11] to RPC 0 at $2.2 \mathrm{GHz}, 2.8 \mathrm{GHz}$ and $3.4 \mathrm{GHz}$, and under the scenario condition defined in Table II (except that PDNR $=24 \mathrm{~dB} \cdot \mathrm{m}^{-2}$ ). The colored horizontal lines and the black circles correspond to the reference $95^{\text {th }}$ percentile of $\Delta a_{\mathrm{RMS}}$ (obtained using RPC 0 only) and the lowest, respectively.

\section{Measurement and Analysis of the Direction FINDING PERFormances USING THE OPTIMAL ADDITIONAL RADIATION PATTERNS}

\section{A. Simulated and measured DF performances with RPC 4}

The simulated and measured DoA estimation accuracy achievable from using the Two Season VA and the MUSIC algorithm are re-evaluated under the scenario reported in Table II, when RPC 4 is added to RPC 0. Desired RPs are obtained from assigning different sets of weighting coefficients to the signals received at the VA ports (see Table I and Table III). Fig. 7 (columns 3 and 4, lines 4 to 6) displays the simulated and measured angular distance $\Delta a_{\mathrm{RMS}}$ at the three frequencies of interest. It can be observed that the accuracy is significantly improved when adding RPC 4 to RPC 0 (see for comparison Fig. 7, columns 3 and 4, lines 1 to 3 ). Moreover, $\Delta a_{\mathrm{RMS}}$ does not exceed $5^{\circ}$ in all directions of the 3D upper half-space within the VA bandwidth. Furthermore, the measurements and simulations results are in good agreement, as it can be confirmed from the values of the maximum and $95^{\text {th }}$ percentile of $\Delta a_{\text {RMS }}$ reported in Table VI. The simulated and measured maximum of $\Delta a_{\mathrm{RMS}}$ are lower than $1.4^{\circ}$ and $2.4^{\circ}$ across the Two Season VA entire bandwidth, respectively. Therefore, using RPC 4 in addition to RPC 0 clearly reduces the measured maximum error of $\Delta a_{\mathrm{RMS}}$ by the following factors: 8.7 at $2.2 \mathrm{GHz}, 27.4$ at $2.8 \mathrm{GHz}$ and 57.8 at $3.4 \mathrm{GHz}$. As for the measured $95^{\text {th }}$ percentile of $\Delta a_{\mathrm{RMS}}$, it is reduced by the following factors: 3.4 at $2.2 \mathrm{GHz}, 3$ at $2.8 \mathrm{GHz}$ and 3.4 at $3.4 \mathrm{GHz}$.

\section{B. Analysis of DF performances}

1) DF performances using RPC 0 only: There are two complementary metrics that can be used to identify the angular areas where the highest values of $\Delta a_{\mathrm{RMS}}$ are reached: (i) the $\mathrm{CRB}$ and (ii) the 3-D ambiguity spectrum (defined in Appendices A-B and A-C). Results are reported in Fig. 7 (columns 1 and 2) at the three frequencies of interest. The computation of the $\mathrm{CRB}_{\phi}$ at $2.2 \mathrm{GHz}$ enables the prediction of the bad estimation in the direction $(\phi, \theta)=\left(40^{\circ}, 50^{\circ}\right)$ (see Figs. 7 (a) and (c)). In the same vein, the angular areas $\left(\theta=68^{\circ}, \phi=\left[30^{\circ} ; 60^{\circ}\right]\right)$ at $2.8 \mathrm{GHz}$ can also be identified (see Figs. 7 (e) and (g)). As for $3.4 \mathrm{GHz}$, the highest values of $\Delta a_{\mathrm{RMS}}$ are found for directions in the angular zones around $\phi=0^{\circ}\left(\bmod 90^{\circ}\right)$, where the risk of angular ambiguity is maximal $\left(\alpha_{\min }\right.$ close to $0^{\circ}$, see Figs. 7 (j) and (k)). It can be observed that the estimation errors at high frequencies result mainly from the angular ambiguity.

2) DF performances using both RPC 0 and RPC 4: As expected by the method used to select the additional RP diversity, RPC 4 improves the CRB values in the entire frequency bandwidth, and it enables the reduction of the estimation errors in the angular areas where the accuracy of the DoA estimation was the lowest. The new CRB values have significantly decreased. Consequently, the highest estimation error in the direction $(\phi, \theta)=\left(40^{\circ}, 50^{\circ}\right)$ at $2.2 \mathrm{GHz}$ is no more apparent thanks to the addition of RPC 4 (see Figs. 7 (c) and (o)). Moreover, the additional RP diversity can also decreases the risk of angular ambiguity. This is actually the case with RPC 4 at the three frequencies of interest, especially at $3.4 \mathrm{GHz}$ (see Figs. 7 (j) and (v)). The risk of ambiguity is significantly reduced when RPC 4 is added, especially in the angular areas around $\phi=0^{\circ}\left(\bmod 90^{\circ}\right)$.

\section{Sensitivity of the Two Season VA}

The sensitivity (defined in Appendix A-E) of the Two Season VA prototype depicted in Fig. 3 is evaluated in the following for a required DoA estimation accuracy of $5^{\circ}$. Therefore, the measured $95^{\text {th }}$ percentile of $\Delta a_{\text {RMS }}$ is displayed in Fig. 8 at the three frequencies of interest and for different PDNR, ranging from $18 \mathrm{~dB} \cdot \mathrm{m}^{-2}$ to $36 \mathrm{~dB} \cdot \mathrm{m}^{-2}$ (with a step of $3 \mathrm{~dB}$ ). It can be observed that the sensitivity of the proposed VA is degraded as the frequency increases when no RPC is added to RPC 0 in the DoA estimation process. When RPC 4 is added to RPC 0 , the sensitivity is improved and remains stable in the upper bandwidth. That yields to the sensitivity of -121 $\mathrm{dBW} \cdot \mathrm{m}^{-2}$ at $2.2 \mathrm{GHz}\left(\mathrm{PDNR} \approx 20 \mathrm{~dB} \cdot \mathrm{m}^{-2}\right),-115 \mathrm{dBW} \cdot \mathrm{m}^{-2}$ at $2.8 \mathrm{GHz}\left(\mathrm{PDNR} \approx 26 \mathrm{~dB} . \mathrm{m}^{-2}\right)$ and $-115 \mathrm{dBW} \cdot \mathrm{m}^{-2}$ at $3.4 \mathrm{GHz}$ $\left(\mathrm{PDNR} \approx 26 \mathrm{~dB} \cdot \mathrm{m}^{-2}\right)$. Indeed, as derived in Section V-B, the addition of RPC 4 to RPC 0 in the DoA estimation process allows the reduction of the ambiguity risk (which increases with frequency). As a consequence, the sensitivity remains stable in the upper operating frequency band. 


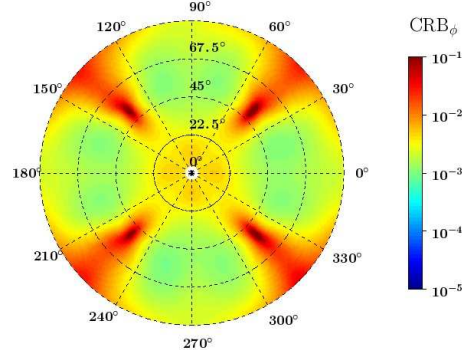

(a)

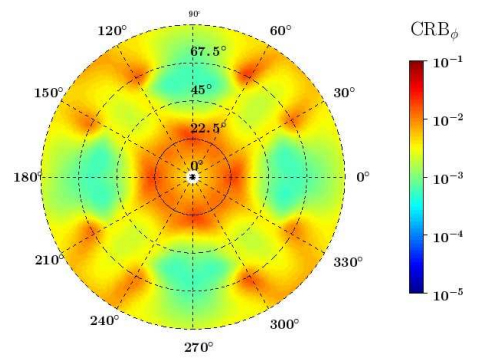

(e)

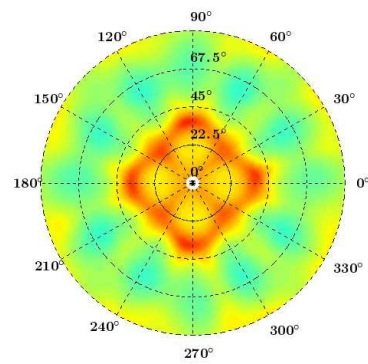

(i)

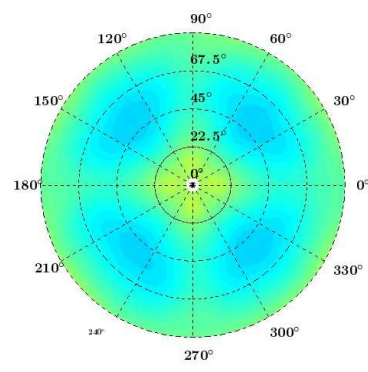

(m)

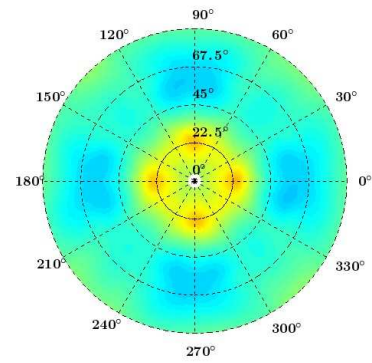

(q)
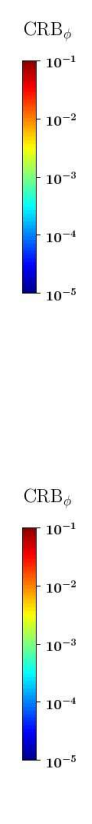

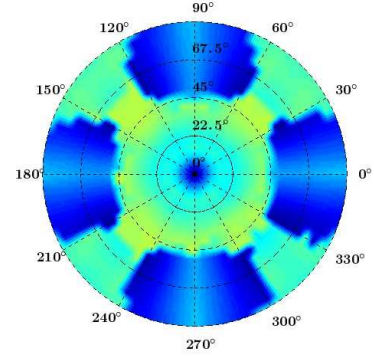

(b)

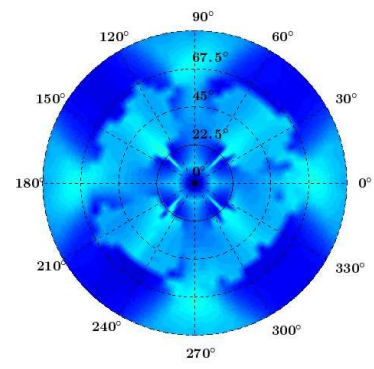

(f)

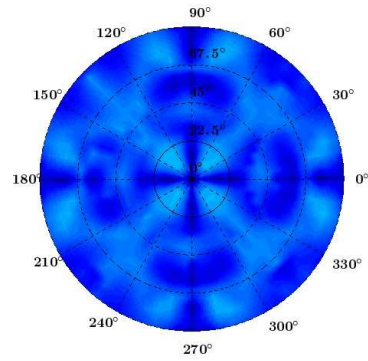

(j)

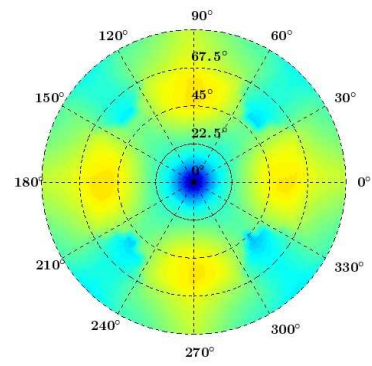

(n)
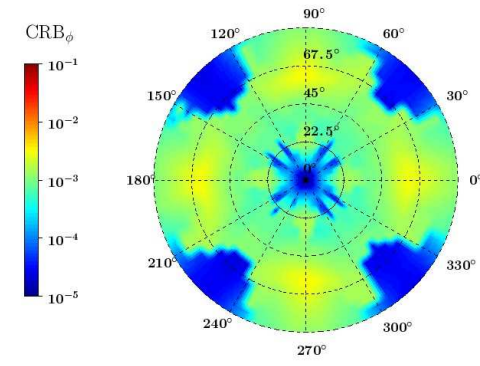

(r)
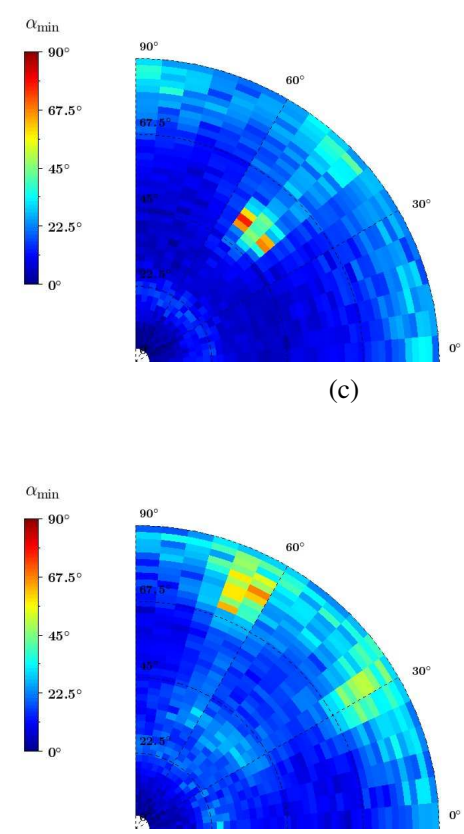

(g)
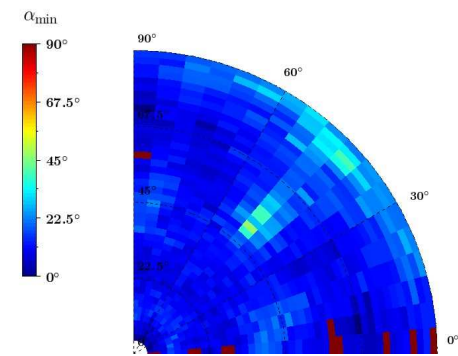

(k)
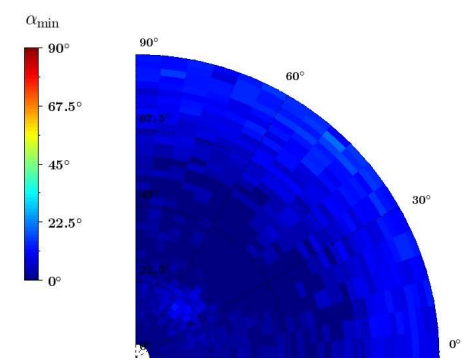

(o)
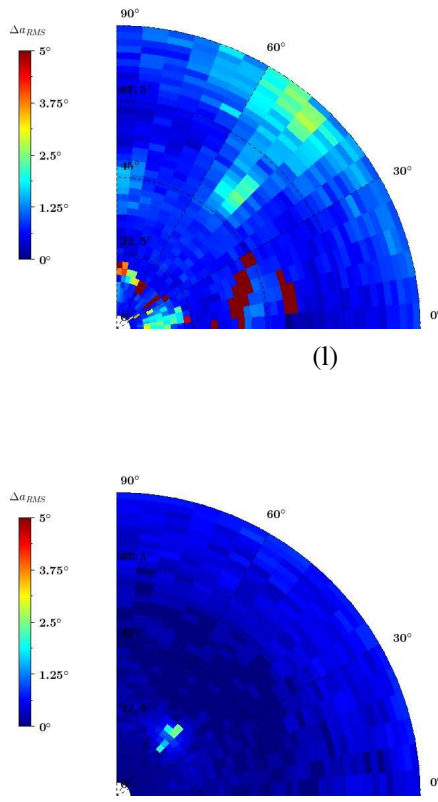

(1)

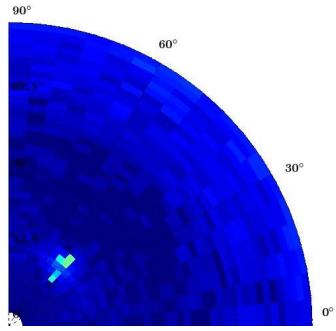

(p)
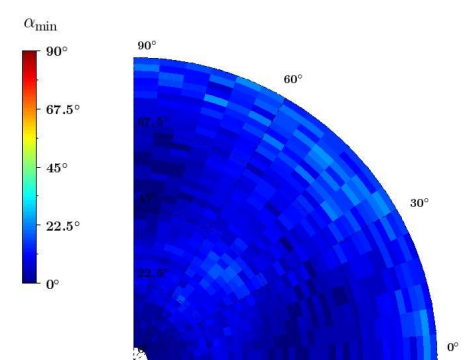

(s)
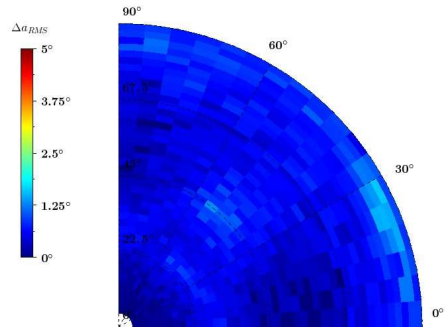

(t)
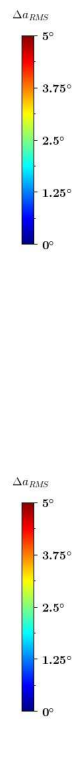


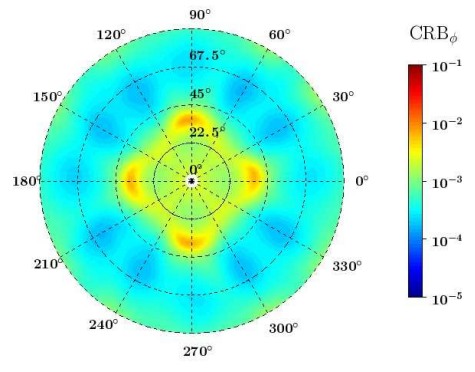

(u)

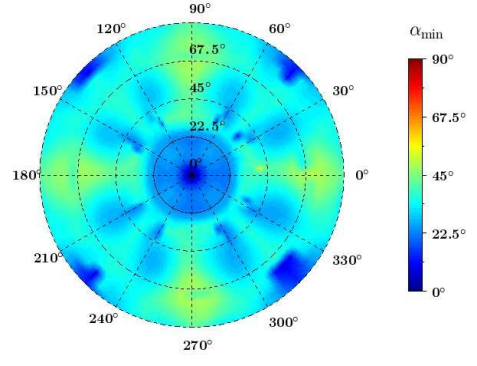

(v)

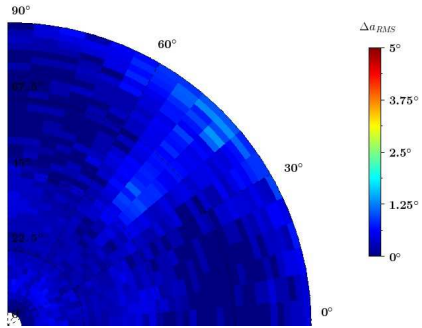

(w)

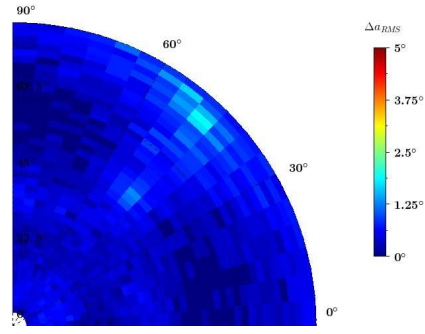

(x)

Fig. 7: $\mathrm{CRB}_{\phi}$ (column 1), $\alpha_{\min }$ (column 2) and $\Delta a_{\mathrm{RMS}}(\phi, \theta)$ (columns 3 and 4) of the Two Season VA from using RPC 0 only (lines 1 to 3) or both RPC 0 and RPC 4 (lines 3 to 6), and under the scenario conditions defined in Table II at the following frequencies: (lines 1 and 4) $2.2 \mathrm{GHz}$, (lines 2 and 5) $2.8 \mathrm{GHz}$, and (lines 3 and 6) $3.4 \mathrm{GHz}$. Columns 1 to 3 and column 4 are simulation results and measurement results, respectively. Elevation angles $\theta$ are given on the radial axis and azimuth angles $\phi$ are given on the angular axis.

TABLE VI: Simulated and measured DF performances from using RPC 0 only or both RPC 0 and RPC 4, and under the scenario conditions defined in Table II (PDNR $\left.=36 \mathrm{~dB} \cdot \mathrm{m}^{-2}\right)$

\begin{tabular}{|c|c|c|c|c|c|c|c|c|}
\hline \multirow{3}{*}{ Frequency } & \multicolumn{4}{|c|}{ RPC 0} & \multicolumn{4}{|c|}{ RPC $0+$ RPC 4} \\
\hline & \multirow{2}{*}{\multicolumn{2}{|c|}{$\begin{array}{cc} & \text { Simulated } \\
\max & 95^{\text {th }} \text { percentile of } \\
\Delta a_{\mathrm{RMS}} & \Delta a_{\mathrm{RMS}}\end{array}$}} & \multirow{2}{*}{\multicolumn{2}{|c|}{$\begin{array}{cc} & \text { Measured } \\
\max & 95^{\text {th }} \text { percentile of } \\
\Delta a_{\mathrm{RMS}} & \Delta a_{\mathrm{RMS}}\end{array}$}} & \multirow{2}{*}{\multicolumn{2}{|c|}{$\begin{array}{cc} & \text { Simulated } \\
\max & 95^{\text {th }} \text { percentile of } \\
\Delta a_{\mathrm{RMS}} & \Delta a_{\mathrm{RMS}}\end{array}$}} & \multirow{2}{*}{\multicolumn{2}{|c|}{$\begin{array}{cc} & \text { Measured } \\
\max & 95^{\text {th }} \text { percentile of } \\
\Delta a_{\mathrm{RMS}} & \Delta a_{\mathrm{RMS}}\end{array}$}} \\
\hline & & & & & & & & \\
\hline $2.2 \mathrm{GHz}$ & $4.2^{\circ}$ & $1.7^{\circ}$ & $20.9^{\circ}$ & $2.4^{\circ}$ & $1.2^{\circ}$ & $0.8^{\circ}$ & $2.4^{\circ}$ & $0.7^{\circ}$ \\
\hline $2.8 \mathrm{GHz}$ & $3.8^{\circ}$ & $2.0^{\circ}$ & $43.9^{\circ}$ & $3.0^{\circ}$ & $1.4^{\circ}$ & $1.1^{\circ}$ & $1.6^{\circ}$ & $1.0^{\circ}$ \\
\hline $3.4 \mathrm{GHz}$ & $142.5^{\circ}$ & $1.6^{\circ}$ & $109.9^{\circ}$ & $3.1^{\circ}$ & $1.4^{\circ}$ & $0.8^{\circ}$ & $1.9^{\circ}$ & $0.9^{\circ}$ \\
\hline
\end{tabular}

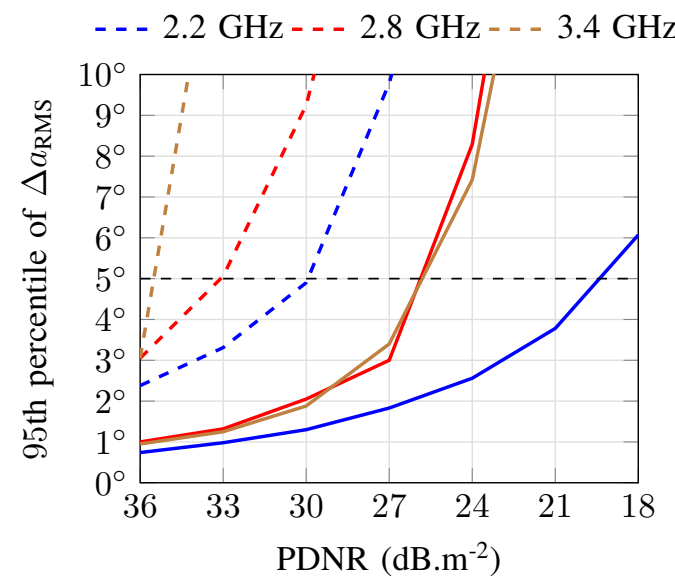

Fig. 8: Measured $95^{\text {th }}$ percentile of $\Delta a_{\mathrm{RMS}}$ for different PDNR using RPC 0 (dashed line), and with RPC 4 added (solid line) in the DoA estimation process at $2.2 \mathrm{GHz}, 2.8 \mathrm{GHz}$ and 3.4 $\mathrm{GHz}$

\section{CONCLUSION}

The DoA estimation performances of a passive, wideband and radiation pattern reconfigurable DF antenna have been reported in this paper. The accurate DoA estimation of an incoming vertically polarized EM field is achieved over a 1.69:1 bandwidth with a 3-D field of view using the MUltiple Signal Classification algorithm. A new method based on the Cramer-Rao lower bound is proposed in order to select the radiation pattern diversity that improves the estimation accuracy across the overall bandwidth. It can be noted that this method is not subject to the DoA estimation technique used for evaluating the DF performances of the vector antenna. Finally, measurement results have been reported to experimentally validate the benefits of radiation pattern diversity offered by a reconfigurable vector antenna. It is demonstrated that such diversity allows a significant improvement of the DoA estimation performances. Next steps will be focused on the benefits of the proposed DF technique to improve the robustness of the direction finder to multipaths, multi-source environment and also, to enhance the estimation accuracy of the polarization parameters of incoming EM fields.

\section{APPENDIX A \\ KEY DESCRIPTORS OF DIRECTION FINDERS}

The estimation performances of any direction finders are subject, on the one hand, to the electrical characteristics of the DF antenna, and on the other hand, to the applied DF technique. The following descriptors will be used in this paper to evaluate the DF performances.

\section{A. Power Density-to-Noise Ratio (PDNR)}

As it is more generic than the classical Signal-to-Noise Ratio (SNR), this parameter (PDNR) is used in this work for describing the operational situation when assessing the DF performances. Indeed, the same value of the SNR cannot be 
associated with all the radiating elements constituting a VA, since their gain differs in the DoA of the incoming EM field. The PDNR is defined as follows

$$
\mathrm{PDNR}=\frac{P_{s}}{P_{n}} .
$$

Moreover, the noise power level at the receiver output is set to $-111 \mathrm{dBm}$, which corresponds to the standard figure of a global system for mobile communications receiver [17].

\section{B. Cramer-Rao lower bound (CRB)}

This parameter gives the lower bound on the accuracy of any unbiased estimator [22]. It allows to define here the theoretical limit of the mean square estimation error that can be reached by a given direction finder. It is important to note that the CRB is independent of the DF technique used. From a technical point of view, the CRB is given by the inverse of the Fischer information matrix $\mathbf{J}$. In this paper, the polarization of the incoming EM field is assumed to be known, and the Gaussian noise is spatially invariant with zero-mean. As a result, the Fischer information matrix $\mathbf{J}$ for estimating the vector parameter $\boldsymbol{\Omega}=\left[\begin{array}{ll}\theta & \phi\end{array}\right]^{\mathrm{T}}$ can be expressed as follows [23]

$$
\mathbf{J}=\left[\begin{array}{ll}
J_{\theta, \theta} & J_{\theta, \phi} \\
J_{\phi, \theta} & J_{\phi, \phi}
\end{array}\right],
$$

with

$$
J_{\boldsymbol{\Omega}_{i}, \boldsymbol{\Omega}_{j}}=\frac{2 N}{\sigma_{n}^{2}} \mathfrak{R e}\left\{\frac{\partial \boldsymbol{d}_{\boldsymbol{c}}{ }^{*}}{\partial \boldsymbol{\Omega}_{i}} \frac{\partial \boldsymbol{d}_{\boldsymbol{c}}}{\partial \boldsymbol{\Omega}_{j}}\right\},
$$

where the $m^{\text {th }}$ component of $\boldsymbol{d}_{\boldsymbol{c}}$, denoted $d_{c}^{m}$, is defined as follows

$$
d_{c}^{m}=\boldsymbol{\beta}^{m} . \boldsymbol{d} .
$$

The study of the CRB consists here to calculate the smallest attainable mean square error for $\theta$ estimation $\left(\mathrm{CRB}_{\theta}\right)$ and $\phi$ estimation $\left(\mathrm{CRB}_{\phi}\right)$ for each DoA in the 3-D upper half-space. The peaks of the CRB correspond to the largest error on $\theta$ or $\phi$ estimations.

\section{Angular Ambiguity Protection (APP)}

The AAP gives the ability of the direction finder to discriminate simultaneously two or more DoAs which are, sufficiently spaced apart with a relative phase-shift multiple of $2 \pi$ and, present co-linear steering vectors [20]. The occurrence of angular ambiguity is related to the spatial distribution of the constitutive radiating elements of the DF antenna. However, it is possible to evaluate the risk of angular ambiguity between two DoAs, sufficiently spaced apart, by verifying whether or not these directions provide two linearly dependent $\left(\phi_{i}, \theta_{i}\right)$ and $\left(\phi_{j}, \theta_{j}\right)$ steering vectors. This verification is performed by analyzing the value of angle $\alpha \in\left[0^{\circ} ; 90^{\circ}\right]\left(\bmod 90^{\circ}\right)$ between the two steering vectors, which is given by [24]

$$
\alpha\left(\phi_{i}, \theta_{i}, \phi_{j}, \theta_{j}\right)=\cos ^{-1}\left(\frac{\left|\boldsymbol{d}_{\boldsymbol{c}}{ }^{*}\left(\phi_{i}, \theta_{i}\right) \cdot \boldsymbol{d}_{\boldsymbol{c}}\left(\phi_{j}, \theta_{j}\right)\right|}{\left\|\boldsymbol{d}_{\boldsymbol{c}}{ }^{*}\left(\phi_{i}, \theta_{i}\right)\right\| \cdot\left\|\boldsymbol{d}_{\boldsymbol{c}}\left(\phi_{j}, \theta_{j}\right)\right\|}\right) .
$$

For $\alpha=0^{\circ}$, different steering vectors are colinear and consequently, the risk of angular ambiguity is very high. Conversely, for $\alpha=90^{\circ}$, the vectors are orthogonal and as a result, there is no a angular ambiguity. In short, the smaller the angle $\alpha$, the greater the risk of angular ambiguity. Therefore, in the directions where $\alpha$ is small, the DoA estimation is more sensitive to the noise of the receiver, and the estimation accuracy is degraded for these angular areas, especially in operational conditions characterized by low PDNR. Moreover, to ensure the best DoA estimation at a fixed PDNR, it is necessary to have simultaneously smaller risk of ambiguity and CRB (in $\theta$ and in $\phi$ ) values. The risk of angular ambiguity in the 3-D half-space is usually evaluated from the 3-D spectrum of the minimum value of $\alpha$ between each pair of angles $\left(\phi_{i}, \theta_{i}, \phi_{j}, \theta_{j}\right)_{i \neq j}[17]$.

\section{DoA estimation accuracy}

This accuracy is the angular error between the estimated angle of arrival and the exact angle. It is conventionally given in terms of the root-mean-square (RMS) value. Depending on the application, this accuracy may be given either as a function of the azimuth or elevation angles, or as a function of the angular distance given by (see, e.g., [25])

$$
\Delta a_{\mathrm{RMS}}(\phi, \theta)=\sqrt{\frac{1}{L} \sum_{i=1}^{L}|\Delta a(\phi, \theta)|^{2}},
$$

where $L$ denotes the number of Monte-Carlo trials and $\Delta a(\phi, \theta)$ designates the angular minimal angular distance between the estimated $(\hat{\phi}, \hat{\theta})$ and actual $(\phi, \theta)$ angles. This angular distance corresponds to the orthodromic distance between two points on the surface of a sphere and is expressed as follows

$$
\begin{aligned}
\Delta a(\phi, \theta)= & \cos ^{-1}(\cos (\theta) \cos (\hat{\theta})+ \\
& \sin (\theta) \sin (\hat{\theta}) \cos (\phi-\hat{\phi})) .
\end{aligned}
$$

The estimation accuracy achieved by the DF antenna over a given angular coverage can be evaluated by analyzing either the maximum value or the $95^{\text {th }}$ percentile of $\Delta a_{\mathrm{RMS}}(\phi, \theta)$ of all estimated DoAs. In this paper, this latter corresponds to the error threshold for respectively $95 \%$ all simulated or measured DoAs in the 3-D half space. Although the highest value of $\Delta a_{\mathrm{RMS}}(\phi, \theta)$ may be interesting in practice, it does not provide a good picture of the antenna performances when the accuracy is very good in the full 3-D space, except in few specific directions. In this case, the $95^{\text {th }}$ percentile of $\Delta a_{\mathrm{RMS}}(\phi, \theta)$ offers a better descriptor as it smooths the estimation errors in these very isolated directions.

\section{E. Sensitivity of the direction finder}

For a given noise power level $P_{n}$, the sensitivity designates the minimum power density $P_{s}$ required at the antenna location to estimate the DoA with an error which does not exceed a prescribed threshold. In other words, it defines the required power at the DF antenna input that ensures a minimum SNR for obtaining the desired accuracy. In this paper, the $95^{\text {th }}$ percentile of the $\Delta a_{\mathrm{RMS}}$ is used to specify the sensitivity of the DF antenna for a maximum error level of $5^{\circ}$. 


\section{ACKNOWLEDGMENT}

The authors would like to thank the French Defense Agency (Direction Général de l'Armement, DGA) and the Occitanie regional council for their financial support. This work is performed in the framework of the AVIAtoR3D project.

\section{REFERENCES}

[1] T. E. Tuncer and B. Friedlander, Classical and Modern Direction-ofArrival Estimation. Academic Press, 2009.

[2] Rohde \& Schwarz, "Antenne ADD253," http://www.rohdeschwarz.com/en/product/add253, visited on Jun. 21, 2018.

[3] CRFS, "DF antenna RFeye Array 300," https://www.crfs.com/allproducts/hardware/direction-finders/array-300/, visited on Jun. 21, 2018.

[4] TCI, "DF antenna Model 643," https://www.tcibr.com/product/tcimodel-643-dual-polarized-vhfuhf-df-and-spectrum-monitoring-antenna/, visited on Jun. 21, 2018.

[5] A. Bellion and C. Le Meins, "Directional multiple-polarization wide band antenna network," Patent, Dec. 21, 2007, US20110133986A1.

[6] L. Scorrano and L. Dinoi, "Experimental characterization of a dualpolarized direction finding array for VHF-UHF frequency bands," in European Conference on Antennas and Propagation (EUCAP), March 2017, pp. 1295-1298.

[7] P. Gething, Radio Direction Finding and Superresolution, ser. Electromagnetics and Radar. P. Peregrinus Ltd., 1991.

[8] E. Ferrara and T. Parks, "Direction finding with an array of antennas having diverse polarizations," IEEE Transactions on Antennas and Propagation, vol. 31, no. 2, pp. 231-236, Mar 1983.

[9] G. F. Hatke, "Performance analysis of the SuperCART antenna array," 1992.

[10] A. Nehorai and E. Paldi, "Vector-sensor array processing for electromagnetic source localization," IEEE Transactions on Signal Processing, vol. 42, no. 2, pp. 376-398, Feb. 1994.

[11] K. T. Wong and X. Yuan, "Vector cross-product direction-finding with an electromagnetic vector-sensor of six orthogonally oriented but spatially noncollocating dipoles/loops," IEEE Transactions on Signal Processing, vol. 59, no. 1, pp. 160-171, Jan 2011.

[12] L. L. Monte, B. Elnour, and D. Erricolo, "Distributed 6D vector antennas design for direction of arrival applications," in International Conference on Electromagnetics in Advanced Applications, Sept 2007, pp. 431-434.

[13] L. L. Monte, B. Elnour, D. Erricolo, and A. Nehorai, "Design and realization of a distributed vector sensor for polarization diversity applications," in International Waveform Diversity and Design Conference, June 2007, pp. 358-361.

[14] M. J. Slater, C. D. Schmitz, M. D. Anderson, D. L. Jones, and J. T. Bernhard, "Demonstration of an electrically small antenna array for UHF direction-of-arrival estimation," IEEE Transactions on Antennas and Propagation, vol. 61, no. 3, pp. 1371-1377, March 2013.

[15] B. Almog, "Compact 3D direction finder," Patent EP20 120184835 , 2013.

[16] A. Musicant, B. Almog, N. Oxenfeld, and R. Shavit, "Vector sensor antenna design for VHF band," IEEE Antennas and Wireless Propagation Letters, vol. 14, pp. 1404-1407, 2015.

[17] J. Lominé, C. Morlaas, C. Imbert, and H. Aubert, "Dual-band vector sensor for direction of arrival estimation of incoming electromagnetic waves," IEEE Transactions on Antennas and Propagation, vol. 63, no. 8, pp. 3662-3671, Aug. 2015.

[18] J. Duplouy, C. Morlaas, H. Aubert, P. Potier, P. Pouliguen, and C. Djoma, "Reconfigurable grounded vector antenna for 3-D electromagnetic direction-finding applications," IEEE Antennas and Wireless Propagation Letters, vol. 17, no. 2, pp. 197-200, Feb 2018.

[19] K. T. Wong, "Direction finding/polarization estimation-dipole and/or loop triad(s)," IEEE Transactions on Aerospace and Electronic Systems, vol. 37, no. 2, pp. 679-684, Apr 2001.

[20] R. Schmidt, "Multiple emitter location and signal parameter estimation," IEEE Transactions on Antennas and Propagation, vol. 34, no. 3, pp. 276-280, Mar. 1986.

[21] S. Chandran, Advances in Direction-of-Arrival Estimation. Artech House, 2005.

[22] P. Stoica and A. Nehorai, "MUSIC, maximum likelihood, and CramerRao bound," IEEE Transactions on Acoustics, Speech, and Signal Processing, vol. 37, no. 5, pp. 720-741, May 1989.

[23] H. V. Trees, Optimum Array Processing: Part IV of Detection, Estimation, and Modulation Theory, ser. Detection, Estimation, and Modulation Theory. Wiley, 2004.
[24] D. Spielman, A. Paulraj, and T. Kailath, "Performance analysis of the MUSIC algorithm," in IEEE International Conference on Acoustics, Speech, and Signal Processing, vol. 11, Apr 1986, pp. 1909-1912.

[25] G. Maral and M. Bousquet, Satellite Communications Systems: Systems, Techniques, and Technology, 5th ed., ser. Communication and Distributed Systems. Wiley, 2009.

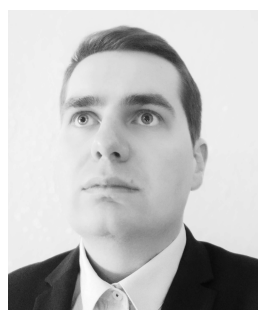

Johan Duplouy received the M.Sc. degree in aeronautical and space telecommunications engineering from ENAC (French Civil Aviation University), Toulouse, France, in 2014, and the Ph.D. degree in electromagnetics from INPT (National Polytechnic Institute), Toulouse, France, in 2019. His Ph.D. focused on wideband and radiation pattern reconfigurable vector antenna for 3-D direction finding application. Since 2019, he is a research engineer, working on antenna and propagation models as well as ElectroMagnetic Compatibility (EMC) solutions for Communication, Navigation, and Surveillance (CNS) systems, on behalf of the French Civil Aviation Directorate.

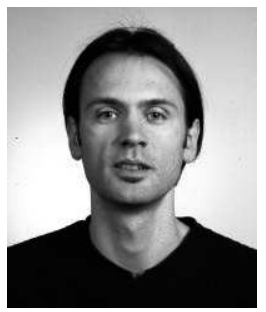

Christophe Morlaas received the Ph.D. degree in electronics from ISAE-SUPAERO ('Institut Supérieur de l'Aéronautique et de l'Espace") in 2000 . He held an antenna system engineer position at ASTRIUM, Toulouse in spatial programs until 2002. Since then, he is an assistant professor with the Electromagnetics and Antennas Research Group of the Telecom lab of ENAC. His research interests include aeronautical communication and navigation systems, electromagnetic modeling, propagation models and antenna design.

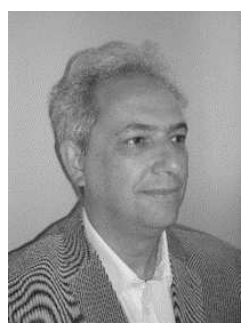

Hervé Aubert (M94, SM99) was born in Toulouse, France, in July 1966. He received the Eng. Dipl. in July 1989 and the Ph.D. degree (with highhonors) in January 1993, both in Electrical Engineering and both from the Institut National Polytechnique (INPT), Toulouse, France. Since February 2001 Herv Aubert is Full Professor at INPT and since January 2015, he is the Head of the Microand Nano-systems for Wireless Communications Research Group at Laboratory for the Analysis and Architecture of Systems (LAAS), National Center for Scientific Research (CNRS), Toulouse, France. Dr. Aubert has performed research works on integral-equation and variational methods applied to electromagnetic wave propagation and scattering. Currently his research activities involve the electromagnetic modelling of complex (multi-scale) structures, and the wireless electromagnetic sensors. He has authored or co-authored 90 papers in indexed journals and 200 communications in International Symposium Proceedings. He holds 8 international patents in the area of antennas and wireless sensors. Dr. Aubert serves as a Subject Editor for Electronics Letters (since 2016), after serving as an Associate Editor for this Journal (2015-2016). He is a member of the editorial board of International Journal of Antennas and Propagation (since 2014). He was the General Chairman of the European Microwave Week in 2015 (Paris, France). 


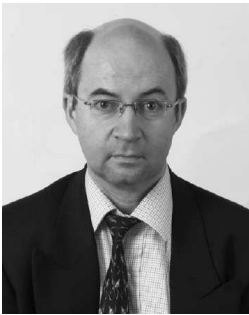

Philippe Pouliguen received the M.S. degree in signal processing and telecommunications, the Doctoral degree in electronic and the "Habilitation á Diriger des Recherches" degree from the University of Rennes 1, France, in 1986, 1990 and 2000. In 1990, he joined the Direction Générale de l'Armement (DGA) at the Centre d'Electronique de l'Armement (CELAR), now DGA Information Superiority (DGA/IS), in Bruz, France, where he was a "DGA senior expert" in electromagnetic radiation and radar signatures. He was also in charge of the EMC (Expertise and ElectoMagnetism Computation) laboratory of DGA/IS. Since December 2009, he is the head of "acoustic and radioelectric waves" scientific domain at DGA, Paris, France. His research interests include electromagnetic scattering and diffraction, Radar Cross Section (RCS) measurement and modeling, asymptotic high frequency methods, radar signal processing and analysis, antenna scattering problems and Electronic Band Gap Materials. In these fields, he has published more than 35 articles in refereed journals and more than 130 conference papers.

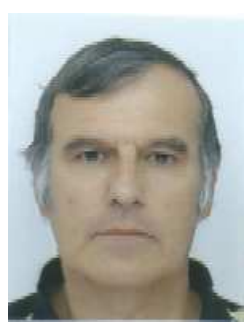

studies and theses.
Patrick Potier received his degree of $\mathrm{Ph}$. D. in structure and property of the material from the University of Rennes, Rennes, France, in 1984. From September 1984 to September 1987, he was engineer of research in Thomson CSF, Paris, France. Since September 1987, he is currently an engineer of Center "Maîtrise de l'information" (Information Superiority) of "Direction Générale de l'armement" (General Armaments Directorate, the French procurement agency). He treats the aspects antennas and radiation, and ensures the follow-up of various 